Manuela Chaves · Jesús García Azorero

\title{
On bifurcation and uniqueness results for some semilinear elliptic equations involving a singular potential
}

\section{Dedicated to Prof. Ambrosetti, with our deepest thanks}

Received September 7, 2005 and in revised form December 8, 2005

Abstract. We present some results concerning the problem

$$
\left\{\begin{array}{l}
-\Delta u=\lambda \frac{u}{|x|^{2}}+u^{q}, \quad u>0 \quad \text { in } \Omega, \\
\left.u\right|_{\partial \Omega}=0,
\end{array}\right.
$$

where $0<q<(N+2) /(N-2), q \neq 1, \lambda \geq 0$ and $\Omega$ is a smooth bounded domain containing the origin. In particular, bifurcation and uniqueness results are discussed.

\section{Introduction}

The paper is organized as follows. In Section 2 we summarize the results concerning existence and bifurcation, in the convex $(q>1)$ and concave $(q<1)$ cases. As we will see, the required a priori estimates become easier in the concave case, due to some general uniqueness results which are not true for the convex problem. Then in Section 3 we prove a uniqueness result for $q>1$, when the domain $\Omega$ is the unit ball. This theorem follows from a careful analysis of the intersections between solutions, and gives a complete picture of the bifurcation diagram in this particular case.

\section{Existence and bifurcation}

A key tool in the study of this kind of problems is the following Hardy inequality:

Lemma 2.1. Assume that $\Omega$ is an open regular subset of $\mathbb{R}^{N}$ and $u \in W_{0}^{1,2}(\Omega)$. Then

$$
\frac{u}{|x|} \in L^{2}(\Omega) \quad \text { and } \quad \lambda_{N} \int_{\Omega} \frac{|u|^{2}}{|x|^{2}} d x \leq \int_{\Omega}|\nabla u|^{2} d x
$$

where $\lambda_{N}=(N-2)^{2} / 4$ is the optimal constant, which is not attained in $W_{0}^{1,2}(\Omega)$.

Research supported by project MTM2004-02223, M.C.Y.T. Spain.

M. Chaves, J. García Azorero: Departamento de Matemáticas, Universidad Autónoma de Madrid, Cantoblanco, 28049 Madrid, Spain; e.mail: manuela.chaves@uam.es, jesus.azorero@uam.es 
The potential $|x|^{-2}$ is too singular to apply directly the classical theorems about bifurcation. From results for problems without singular potential (see for instance [16]), one would expect bifurcation from infinity in the sublinear case $0<q<1$, and bifurcation from the trivial solution in the superlinear case $1<q<(N+2) /(N-2)$. However, as shown in [9], this is not true, and bifurcation from infinity also occurs in the superlinear case. In the next subsections we will sketch the main steps of the proof of this result. A crucial role is played by the following nonexistence result:

Lemma 2.2. Let $\lambda=\lambda_{N}$. Then problem (1) has no solution $u \in W_{0}^{1,2}(\Omega), u>0$.

This result is true even in the sense of distributions when $\lambda>\lambda_{N}$. It can be deduced from the Baras-Goldstein [4] nonexistence result in the parabolic case.

Remark 2.3. In this paper we are mainly concerned with bifurcation results in the Sobolev space $W_{0}^{1,2}(\Omega)$. When $q>1$, a complete discussion about existence and nonexistence of solutions in the sense of distributions, in the radial case, can be found in [6]. It is proved that for each $\lambda \in\left(0, \lambda_{N}\right)$ there exists an exponent $p^{+}(\lambda)>(N+2) /(N-2)$ such that if $1<q<p^{+}(\lambda)$ then there exists a nontrivial solution in the sense of distributions, and if $q \geq p^{+}(\lambda)$ then the only positive distributional solution is $u \equiv 0$.

\subsection{Truncation}

The natural idea is to pass to the limit in the truncated problems

$$
-\Delta u=\lambda W_{n}(x) u+u^{q}, \quad u \geq 0, \quad x \in \Omega,\left.\quad u\right|_{\partial \Omega}=0,
$$

where $W_{n}(x)=\min \left\{n,|x|^{-2}\right\}$. Since the potential is bounded, we can use general bifurcation results, getting the existence of branches of solutions $\Gamma_{n}$ which bifurcate from the first eigenvalue of the corresponding linear problem, and satisfy some a priori bounds. First, it is easy to see the following:

Lemma 2.4. Consider the first eigenvalue $\lambda_{1}(n)$ of the problem

$$
\begin{cases}-\Delta \psi_{1}=\lambda W_{n}(x) \psi_{1}, & x \in \Omega \subset \mathbb{R}^{N}, \\ \psi_{1}(x)=0, & x \in \partial \Omega,\end{cases}
$$

Then $\lambda_{1}(n) \geq \lambda_{N}$, and $\lim _{n \rightarrow \infty} \lambda_{1}(n)=\lambda_{N}$.

Next, to pass to the limit some kind of compactness is needed. Notice that if we consider the energy functional associated to the truncated problem

$$
J_{n}(u, \lambda)=\frac{1}{2} \int_{\Omega}|\nabla u|^{2}-\frac{\lambda}{2} \int_{\Omega} W_{n}(x) u^{2} d x-\frac{1}{q+1} \int_{\Omega} u^{q+1} d x,
$$

it can be proved that sequences of solutions of the truncated problems are Palais-Smale sequences for the limit energy functional. Moreover, the limit energy functional satisfies 
the Palais-Smale condition if $\lambda<\lambda_{N}$ (some care is needed in the proof of this PalaisSmale condition; details can be seen in [10] and [11]). So, it is in this region that we will be able to pass to the limit.

If we look at the geometry of these energy functionals, the behaviour is completely different if $q<1$ or $q>1$; so each case must be analyzed separately. Before performing this analysis, we state an important common property to both cases, the following blow-up result:

Lemma 2.5. Let $\left\{u_{n}\right\}_{n \in \mathbb{N}}$ be a sequence of solutions of 2 for $\lambda=\lambda_{N}$. Then $\left\|u_{n}\right\|_{W_{0}^{1,2}(\Omega)}$ $\rightarrow \infty$ as $n \rightarrow \infty$.

Proof. Just a comment about the proof of this lemma: the main ingredient is an improved Hardy inequality (see (4.10) in [7]), which can be stated as follows. Given $1<p<2 *$, there exists a constant $\alpha_{p}>0$ such that for any $u \in W_{0}^{1,2}(\Omega), u \neq 0$,

$$
\frac{\int_{\Omega}|\nabla u|^{2}-\lambda_{N} \int_{\Omega}|x|^{-2} u^{2}}{\left(\int_{\Omega} u^{p}\right)^{2 / p}} \geq \alpha_{p} .
$$

This inequality implies that the sequence $J_{n}\left(u_{n}, \lambda_{N}\right)$ does not converge to zero, and then an easy contradiction argument concludes the proof.

\subsection{Sublinear case, $0<q<1$}

This case becomes simpler thanks to the following powerful comparison result, which says that in the sublinear case sub- and supersolutions are always ordered (for the proof, see [2]):

Lemma 2.6. Assume that $f$ is a continuous function such that $f(u) / u$ is decreasing. Let $u, v \in W_{0}^{1,2}(\Omega)$ be such that

$$
\begin{array}{lll}
-\Delta u-\lambda W_{n}(x) u \geq f(u), & u>0 \quad \text { in } \Omega, & \left.u\right|_{\partial \Omega}=0 \\
-\Delta v-\lambda W_{n}(x) v \leq f(v), & v>0 \quad \text { in } \Omega, & \left.v\right|_{\partial \Omega}=0
\end{array}
$$

Then $u \geq v$ in $\Omega$. The same result holds for $W_{\infty}(x)=|x|^{-2}$.

This comparison lemma implies a uniqueness result in the case $0<q<1$, which will be often used in what follows.

For each truncated problem (2) we can apply the classical results about bifurcation from infinity (see Ambrosetti-Hess [3] or Rabinowitz [17]), getting a global branch of positive solutions $\Gamma_{n} \subset W_{0}^{1,2}(\Omega) \times \mathbb{R}$ that meets $\left(\infty, \lambda_{1}(n)\right)$ from the left; moreover, this is the unique point of bifurcation from infinity for problem 2 . Since the problem with $\lambda=0$ has a unique positive solution, $v_{0}$, all these branches pass through the point $\left(v_{0}, 0\right)$. To pass to the limit we use the following topological lemma (see [20]): 
Lemma 2.7. Let $\Gamma_{n}$ be a sequence of connected closed sets of a complete metric space such that $\lim \inf \Gamma_{n} \neq \emptyset$ and $\bigcup_{n \in \mathbb{N}} \Gamma_{n}$ is relatively compact. Then $\lim \sup \Gamma_{n}$ is a connected nonempty set.

In our case, since the lim inf of the set of branches is not empty, because, by uniqueness, all these branches cross the axis $\lambda=0$ at the same point, and the Palais-Smale condition provides the compactness, we get:

Theorem 2.8. Let $0<q<1$. Then there exists an unbounded continuum of positive solutions, $\Gamma \subset W_{0}^{1,2}(\Omega) \times\left[0, \lambda_{N}\right)$, which passes through the point $\left(v_{0}, 0\right)$, v $v_{0}$ being the unique positive solution to problem (1) for $\lambda=0$, and blows up as $\lambda \nearrow \lambda_{N}$.

Moreover, the limit branch is nondegenerate. The approximate branches do not collapse to the vertical axis; this follows from the uniqueness result for $\lambda=0$ : let $\left\{u_{n}\right\}$ be a sequence of solutions of the truncated problems such that $\lambda_{n} \rightarrow 0$. Then the Palais-Smale condition implies that $u_{n} \rightarrow v_{0}$, where $v_{0}$ is the solution corresponding to the limit problem when $\lambda=0$.

On the other hand, the approximate branches are bounded away from the horizontal axis, since by uniqueness solutions correspond to minima of the energy functional and so

$$
J_{n}\left(u_{n}, \lambda_{n}\right)=\min J\left(u, \lambda_{n}\right) \leq \min \left(\frac{1}{2} \int_{\Omega}|\nabla u|^{2}-\frac{1}{q+1} \int_{\Omega} u^{q+1}\right)=-C_{q}<0 .
$$

Finally, an easy rescaling argument shows that the unique point of bifurcation from infinity for the limit problem is $\lambda_{N}$.

\subsection{Superlinear case $1<q<(N+2) /(N-2)$}

In this situation we can apply the global bifurcation theorem by Rabinowitz for each truncated problem, finding an unbounded branch, $\Gamma_{n}$, bifurcating to the left from $\left(\lambda_{1}(n), 0\right)$, and crossing the axis $\lambda=0$. It is well known that in general for problems with superlinear growth nonuniqueness of positive solution is possible. This makes a great difference with the sublinear case, since most of the a priori bounds that we use there are not valid now. Anyway, passing to the limit through the set of branches of the truncated problems we get

Theorem 2.9. Let $1<q<(N+2) /(N-2)$. Then there exists an unbounded continuum of positive solutions, $\Gamma \subset W_{0}^{1,2}(\Omega) \times\left[0, \lambda_{0}\right), \lambda_{0} \leq \lambda_{N}$, passing through the point $(v, 0)$, $v$ being a solution to problem (1) for $\lambda=0$.

Proof. When $\lambda=0$, we have a uniform a priori bound for the solutions (see GidasSpruck [13]); hence, as for $\lambda=0$ the trivial solution is isolated, taking a subsequence if necessary we can assume that all the branches, $\Gamma_{n}$, corresponding to the approximate problems contain the same point $(v, 0)$. Therefore, since the Palais-Smale condition holds if $0 \leq \lambda<\lambda_{N}$, we can pass to the limit. Moreover it is easy to see that the continuum that we found in the limit is nondegenerate, since the improved Hardy inequality implies that 
the branch is bounded away from the horizontal axis (see [10]). In fact, given $\lambda \in\left[0, \lambda_{N}\right)$, if we have a solution $u_{\lambda}$, multiplying by $u_{\lambda}$ in the equation and integrating by parts we get

$$
\int u_{\lambda}^{q+1} d x=\int\left(\left|\nabla u_{\lambda}\right|-\frac{\lambda}{|x|^{2}} u_{\lambda}^{2}\right) d x \geq \alpha_{q}\left(\int u_{\lambda}^{q+1} d x\right)^{2 /(q+1)} .
$$

Since $q>1$, we have $\int u_{\lambda}^{q+1} d x>C>0$ uniformly in $\lambda \in\left[0, \lambda_{N}\right)$. On the other hand, it follows from Lemma 2.5 that the limit branch cannot remain bounded in the interval $\left(0, \lambda_{N}\right)$.

Notice that the continuum that we found is unbounded, it cannot cross the vertical line $\left\{\lambda=\lambda_{N}\right\}$, and when $\lambda=0$ the set of solutions is bounded. Then we can deduce the existence of a point of bifurcation from infinity, $\lambda_{0} \leq \lambda_{N}$. This bifurcation from infinity result is a consequence of the singularity of the potential $|x|^{-2}$ : if we consider a potential $|x|^{-\alpha}$ with $\alpha<2$, the branch of solutions bifurcates from the trivial solution when $\lambda$ equals the first eigenvalue of the corresponding problem. Then a natural question is to ask if $\lambda_{0}=\lambda_{N}$. It is not hard to see that this is the case if the geometry of the domain $\Omega$ implies that we have a unique solution. In fact, if the solution is unique, then it comes from the Mountain Pass Lemma of Ambrosetti-Rabinowitz, and then we can get some useful a priori estimates. Suppose by contradiction that there exists $\lambda_{0}<\lambda_{N}$ and sequences $\lambda_{n} \rightarrow \lambda_{0}$ and $\left\{u_{n}\right\}$ such that $\left\|u_{n}\right\| \rightarrow \infty$. Then

$$
J\left(u_{n}, \lambda_{n}\right)=\inf _{\gamma} \sup _{t} J\left(\gamma(t), \lambda_{n}\right) \leq \inf _{\gamma} \sup _{t} J(\gamma(t), 0) \equiv C_{q}
$$

where the infimum is taken over the set of continuous paths joining the origin to a fixed $z_{0} \in W_{0}^{1,2}(\Omega)$ such that $J\left(z_{0}, 0\right)<0$. On the other hand,

$$
\begin{aligned}
C_{q} \geq J\left(u_{n}, \lambda_{n}\right) & =J\left(u_{n}, \lambda_{n}\right)-\frac{1}{q+1}\left\langle J^{\prime}\left(u_{n}, \lambda_{n}\right), u_{n}\right\rangle \\
& \geq\left(\frac{1}{2}-\frac{1}{q+1}\right)\left(1-\frac{\lambda_{n}}{\lambda_{N}}\right) \int_{\Omega}\left|\nabla u_{n}\right|^{2} d x
\end{aligned}
$$

And this is impossible since $\lambda_{0}=\lim \lambda_{n}<\lambda_{N}$.

Finding general conditions on the domain $\Omega$ such that for $0<\lambda<\lambda_{N}$ uniqueness of positive solution holds seems to be an interesting (and nontrivial) open problem. In the next section we will prove uniqueness when $\Omega$ is a ball.

\section{Uniqueness}

The classical regularity theory for elliptic equations does not work in this kind of problems because of the singularity of the potential $|x|^{-2}$. In fact, solutions to problem (1) are unbounded at the origin: 
Lemma 3.1. Let $u \in W_{0}^{1,2}\left(B_{1}(0)\right)$ be a positive solution to

$$
\left\{\begin{array}{l}
-\Delta u=\frac{\lambda}{|x|^{2}} u+u^{q}, \quad x \in B_{1}(0), \\
\left.u\right|_{\partial B_{1}}=0
\end{array}\right.
$$

Then $u \notin L^{\infty}\left(B_{1}(0)\right)$.

Proof. Fix $\epsilon$ and $\delta$ in such a way that $-\Delta u-\left(\lambda /|x|^{2}\right) u>\epsilon$ if $|x|<\delta$. Then the proof is by a comparison argument, taking as a test function $\phi(x)=c \omega(x / \delta)$, where $c$ is a small constant (which depends on $\epsilon, \delta, \lambda$ and $N$ ) and $\omega(x)=|x|^{-(N-2) / 2+\sqrt{\lambda_{N}-\lambda}}-|x|^{2}$.

The existence of solutions for $\lambda<\lambda_{N}$ follows from variational arguments. In fact, taking into account that the Hardy inequality allows us to work in the usual Sobolev spaces, we can see that the geometry of the energy functional satisfies the hypotheses of the classical Mountain Pass Theorem, and the Palais-Smale condition holds for $\lambda<\lambda_{N}$. The uniqueness seems to be delicate, because the usual techniques like Pohozaev type energy estimates or shooting arguments fail since the solutions are unbounded. See for instance [15], [22], [21], where uniqueness results for some elliptic problems with bounded solutions are proved. The main theorem, whose proof will be developed in the next subsections, is the following:

Theorem 3.2. Let $N \geq 3,1<q<(N+2) /(N-2)$, and $0<\lambda<\lambda_{N} \equiv(N-2)^{2} / 4$. Then problem 5 ) has a unique positive solution $u \in W_{0}^{1,2}\left(B_{1}(0)\right)$.

As a consequence of this theorem, taking into account the bifurcation results in the previous section, we can deduce the following:

Theorem 3.3. There exists an unbounded continuum of positive solutions to problem (5), $\Gamma \subset W_{0}^{1,2}\left(B_{1}(0)\right) \times\left[0,(N-2)^{2} / 4\right)$, passing through the point $(u, 0), u$ being the unique solution to problem (5) for $\lambda=0$.

\subsection{Radial symmetry}

The first step in the proof is a symmetry result. This theorem (which follows readily from the arguments in [19]) holds true under very general hypotheses. In fact, if we give a meaning to the equation in the distributional setting, namely if we assume that $u \in L_{\text {loc }}^{1}$ and $\left(\lambda /|x|^{2}\right) u+u^{q} \in L_{\text {loc }}^{1}$, then the nonlinearity of the right hand side implies that $u \in L_{\text {loc }}^{q}$ with $q>1$. But if we avoid a neighbourhood of the origin, it is also true that $\left(\lambda /|x|^{2}\right) u \in L_{\text {loc }}^{q}\left(B_{1}(0) \backslash\{0\}\right)$ and hence $u \in W_{\text {loc }}^{1,2}\left(B_{1}(0) \backslash\{0\}\right)$.

Theorem 3.4. Let $u \in W_{\mathrm{loc}}^{1,2}\left(B_{1}(0) \backslash\{0\}\right)$ be a positive solution to problem 5 . Then $u$ is radially symmetric about the origin. 
Proof. This symmetry result follows from the well known moving planes method (see [12], [18], [5]), as used by Terracini [19] in a similar problem in $\mathbb{R}^{N}$ involving the critical potential $|x|^{-2}$.

In the radial setting, $u=u(r), r=|x|$, problem (5) takes the form

$$
u_{r r}+\frac{N-1}{r} u_{r}+u^{q}+\frac{\lambda}{r^{2}} u=0, \quad u>0 \quad \text { for } 0<r<1, \quad u(1)=0,
$$

with the additional condition $u \in W_{0}^{1,2}\left(B_{1}(0)\right)$. A first elementary estimate of the behaviour of solutions to 6 close to the origin is the following:

Lemma 3.5. Let $u(r) \in W_{0}^{1,2}\left(B_{1}(0)\right)$ be a solution of 6 . Then

$$
\lim _{r \rightarrow 0} r^{\alpha} u(r) \rightarrow 0 \quad \text { as } r \rightarrow 0, \text { for every } \alpha>\frac{N-2}{2} .
$$

Proof. Notice that by elliptic regularity, energy solutions $u \in W_{0}^{1,2}\left(B_{1}(0)\right)$ to problem 6) are regular away from the origin, and therefore, given $r>0$, we can write

$$
\begin{aligned}
u(r) & =-\int_{r}^{1} u^{\prime}(s) d s=-\int_{r}^{1} u^{\prime}(s) s^{(N-1) / 2} s^{-(N-1) / 2} d s \\
& \leq\left(\int_{r}^{1}\left|u^{\prime}(s)\right|^{2} s^{N-1} d s\right)^{1 / 2}\left(\int_{r}^{1} s^{-(N-1)} d s\right)^{1 / 2} \leq C r^{-(N-2) / 2} .
\end{aligned}
$$

Moreover, since solutions to equation (6) are regular in $(0,1)$, it is obvious that they cannot have any interior local minima (recall that $\lambda>0$ ); therefore any positive solution $u(r)$ is unbounded and decreasing. Let us point out that the usual uniqueness proofs by shooting type arguments do not work in this case, since the solutions are unbounded at the origin. And, moreover, an alternative shooting proof starting from $r=1$ presents an extra difficulty, since as we will see, for some values of the parameters $\lambda$ and $q$, problem (5) has multiple unbounded non-energy solutions. Instead of these approaches, our proof is based on the study of the number of intersections between any two different solutions of the problem.

\subsection{First transformation: phase plane analysis}

Taking into account the homogeneity of the equation, it is convenient to introduce, as in [19], the new variables

$$
\psi(s)=e^{\alpha s} u\left(e^{s}\right), \quad \alpha=\frac{2}{q-1}, \quad r=e^{s},
$$

in such a way that $(6)$ takes the autonomous form

$$
\psi_{s s}+h \psi_{s}+\psi^{q}+k \psi=0, \quad \psi>0 \text { for } s<0, \psi(0)=0
$$



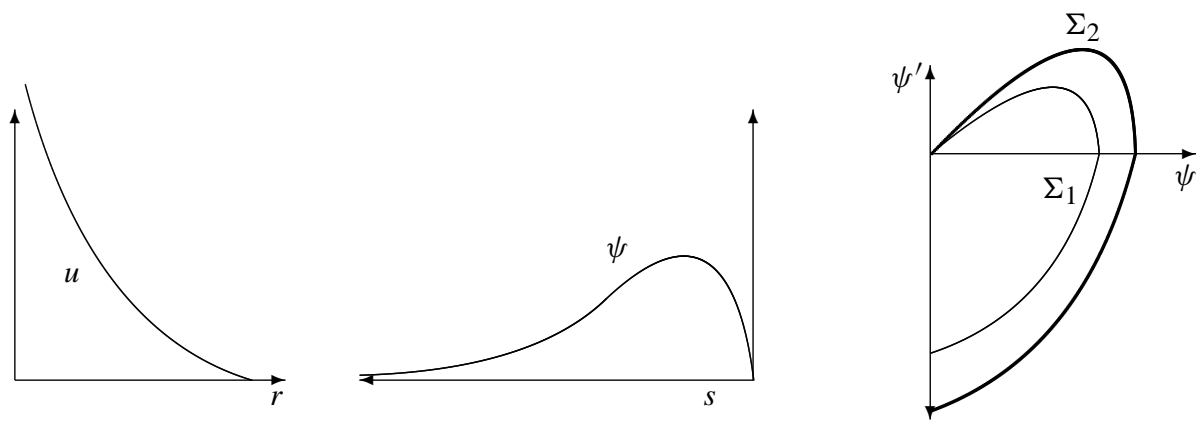

Fig. 1. First change of variables and trajectories in the phase plane.

with $h=N-2-2 \alpha$ and $k=\lambda-h \alpha-\alpha^{2}$. On the other hand, in the new variables the limit condition (7) obtained in Lemma 3.5 implies

$$
\lim _{s \rightarrow-\infty} \psi(s)=0 .
$$

We note that in our range of parameters, the constant $h$ is always negative, and moreover, the dependence on $\lambda$ is hidden in the parameter $k$. We have

$$
\left\{\begin{array}{l}
\psi^{\prime}=w, \\
w^{\prime}=-h w-k \psi-\psi^{q} .
\end{array}\right.
$$

We work in the region $\psi \geq 0$, and we are looking for the orbits which connect the critical point $(0,0)$ and a point of the type $(0, c)$ for some negative value of $c$ (see Fig. 1). To study the local behaviour near the critical point $(0,0)$, we linearize $[11)$ around this point, getting

$$
\left\{\begin{array}{l}
\psi^{\prime}=w, \\
w^{\prime}=-h w-k \psi .
\end{array}\right.
$$

The corresponding eigenvalues are given by

$$
\mu_{ \pm}=-h / 2 \pm \sqrt{h^{2} / 4-k}=-h / 2 \pm \sqrt{(N-2)^{2} / 4-\lambda}
$$

and the associated eigenvectors are $\mathbf{e}_{ \pm}=\left(1, \mu_{ \pm}\right)$. In particular, taking into account the change of variables, the eigenvalues give just the rate of the singularity that the function $u$ has at the origin; this point will be carefully analyzed below. Concerning the critical points, we recall that the constant $h$ is negative and $h^{2} / 4-k>0$. Hence, the critical point $(0,0)$ is a saddle point if $k<0$, while it is an unstable node if $k>0$, and a degenerate unstable node if $k=0$. On the other hand, when $k<0$, there is a second critical point $\left(|k|^{1 /(q-1)}, 0\right)$. Linearization around this point shows that it is unstable, and therefore the general theory of ODEs gives us the following result.

Proposition 3.6. Assume that $k<0$. Then there exists a unique unstable manifold which leaves the origin along the direction $\mathbf{e}_{+}$, as well as a unique stable manifold entering along the vector $\mathbf{e}_{-}$. 
Hence, the desired uniqueness result follows in a straightforward way if $k$ is negative, or, in terms of the original parameters,

$$
\lambda<\frac{2(N-2)}{q-1}-\frac{4}{(q-1)^{2}} \quad\left(<\lambda_{N}\right) .
$$

Remark 3.7. Notice that the case $k<0$, in the notation of [6], corresponds to the range $q \in\left(p^{-}(\lambda),(N+2) /(N-2)\right) \subset\left(p^{-}(\lambda), p^{+}(\lambda)\right)$. In this range the authors of [6] exhibit an explicit solution $u(r)=A r^{-2 /(q-1)}$. This singular explicit solution is related to the second critical point $\left(|k|^{1 /(q-1)}, 0\right)$; see Lemma 3.11.

As mentioned above, this analysis does not give any information about uniqueness of the orbits leaving the origin of the system for $k \geq 0$ (in this case we should use, for instance, some version of the Hartman-Grobman theorem, see [14], which requires a nonresonance condition which fails for some values of $\lambda$ ). This is the reason why we introduce a different approach to the problem. We begin by obtaining a more precise estimate for the singularity of our solutions.

Lemma 3.8. Suppose $k>0$. Let $\psi_{+}$(respectively $\psi_{-}$) be an arbitrary solution of (9) such that the corresponding orbit in the phase plane $(\psi, w)$ leaves the origin along the direction $\mathbf{e}_{+}$(respectively $\left.\mathbf{e}_{-}\right)$. Then there exists a positive constant $C_{ \pm}=C\left(\psi_{ \pm}\right)$such that

$$
\lim _{s \rightarrow-\infty} e^{-\mu_{ \pm} s} \psi_{ \pm}(s)=C_{ \pm}
$$

Proof. We only deal with solutions of the type $\psi_{+}$, since similar arguments apply to solutions of the type $\psi_{-}$, and we omit the subscript + in what follows. We refer to [8] for the complete details. On the one hand, it follows from the phase plane analysis above that

$$
\psi(s) \equiv e^{\mu s+o(s)} \quad \text { as } s \rightarrow-\infty .
$$

Define $H(s)=\mu \psi(s)-\psi^{\prime}(s)$. By using equation 9 , we find that $\psi$ and $H$ satisfy

$$
\left\{\begin{array}{l}
\psi^{\prime}=\mu \psi-H \\
H^{\prime}=-(\mu+h) H+\psi^{q}
\end{array}\right.
$$

After multiplying by appropriate factors in both equations and integrating we obtain

$$
e^{-\mu s} \psi(s)=\int_{s}^{0} e^{-(2 \mu+h) t}\left[-\psi^{\prime}(0)-\int_{t}^{0} e^{(\mu+h) r} \psi^{q}(r) d r\right] d t .
$$

Next we prove that

$$
G(s) \equiv-\psi^{\prime}(0)-\int_{s}^{0} e^{(\mu+h) r} \psi^{q}(r) d r \rightarrow 0 \quad \text { as } s \rightarrow-\infty .
$$

By (14) and the definitions of $\mu$ and $h$ the above limit exists and is finite. Denote it by $l$ and assume that $l \neq 0$. Since $-(2 \mu+h)<0$, we have

$$
\int_{s}^{0} e^{-(2 \mu+h) t}\left[-\psi^{\prime}(0)-\int_{t}^{0} e^{(\mu+h) r} \psi^{q}(r) d r\right] d t \rightarrow \infty \quad \text { as } s \rightarrow-\infty,
$$


Hence by using L'Hospital's rule we have for every $\varepsilon>0$ small enough,

$$
e^{\varepsilon s} \int_{s}^{0} e^{-(2 \mu+h) t}\left[-\psi^{\prime}(0)-\int_{t}^{0} e^{(\mu+h) r} \psi^{q}(r) d r\right] d t \rightarrow \infty \quad \text { as } s \rightarrow-\infty,
$$

a contradiction with (14), which proves (17). Finally, it is not difficult to check that the function $F(s)=e^{-(2 \mu+h) s} G(s)$ is integrable in the interval $(-\infty, 0)$. In fact, from 14 and L'Hospital's rule we get

$$
e^{-\varepsilon s} F(s) \equiv e^{-(2 \mu+h+\varepsilon) s}\left[-\psi^{\prime}(0)-\int_{s}^{0} e^{(\mu+h) r} \psi^{q}(r) d r\right] \rightarrow 0 \quad \text { as } s \rightarrow-\infty,
$$

for every $\varepsilon>0$ small enough and hence the function $F$ is integrable in $(-\infty, 0)$. We also note that $G(s)$ is increasing. This implies, by using $(17)$, that $G(s)$ is positive and the same holds for $F(s)$. The result follows since $e^{-\mu s} \psi(s)=\int_{s}^{0} F(t) d t$.

Remark 3.9. After translating the information to the original variables $u(r)$, we see that the solutions to problem (6) are related to the orbits corresponding to the eigenvectors $\mathbf{e}_{ \pm}$

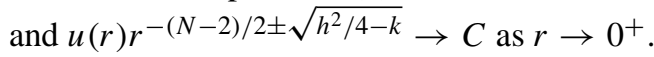

This means that solutions associated to the eigenvalue $\mu_{+}$belong to the Sobolev space $W_{0}^{1,2}\left(B_{1}(0)\right)$, while solutions associated to the eigenvalue $\mu_{-}$are too singular. Hence, we will distinguish between energy (or regular) solutions, and singular solutions. We also summarize the results concerning the analysis of the singularity for the other values of the parameter $k$. The proofs can be found in [8].

Lemma 3.10. Suppose $k=0$. Then there exists a positive constant $C$ such that

$$
\lim _{s \rightarrow-\infty} e^{-\mu_{+} s} \psi_{+}(s)=C \quad \text { and } \quad \lim _{s \rightarrow-\infty}|s|^{1 /(q-1)} \psi_{-}(s)=\left(\frac{-h}{q-1}\right)^{1 /(q-1)} .
$$

Lemma 3.11. Suppose $k<0$. Then there exists a positive constant $C$ such that

$$
\lim _{s \rightarrow-\infty} e^{-\mu_{+} s} \psi_{+}(s)=C \quad \text { and } \quad \lim _{s \rightarrow-\infty} \psi_{-}(s)=|k|^{1 /(q-1)} .
$$

Remark 3.12. Notice that energy solutions always behave as $r^{-(N-2) / 2+\sqrt{\lambda_{N}-\lambda}}$.

\subsection{Estimate of the number of intersections}

Given two solutions $\psi_{1}(s)$ and $\psi_{2}(s)$ of $(9)-(10)$, we denote by $I\left(\psi_{1}, \psi_{2}\right)$ the number of intersections of these functions, that is, the number of sign-changes of the difference $\psi_{1}(s)-\psi_{2}(s)$. The next results are valid for any value of $k \in \mathbb{R}$ and for any two solutions to problem 9 .

Proposition 3.13. Let $\psi_{1}(s)$ and $\psi_{2}(s)$ be solutions of $(9)$, with $N \geq 3,1<q<$ $(N+2) /(N-2)$, and $0<\lambda<(N-2)^{2} / 4$. Then $I\left(\psi_{1}, \psi_{2}\right) \leq 1$. 

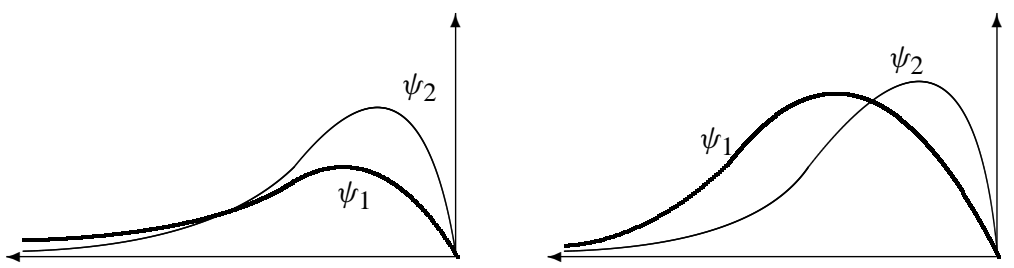

Fig. 2. Admissible intersections

Proof. Consider the phase plane of equation (9) and the corresponding orbits satisfying the associated system (11). Denote by $\Sigma_{i}=\left\{\left(\psi_{i}(s), w_{i}(s)\right): s<0\right\}, i=1,2$, the orbits corresponding to the solutions $\psi_{1}$ and $\psi_{2}$ respectively and assume that

$$
\psi_{2}^{\prime}(0)<\psi_{1}^{\prime}(0) .
$$

(See the diagram in Fig. 1.) Let $S$ be the closed connected domain in the half plane $\psi \geq 0$, bounded by the orbit $\Sigma_{1}$ and the straight line $\psi \equiv 0$. Assume now that $I\left(\psi_{1}, \psi_{2}\right)>1$ and denote by $s_{i}^{*}<0, i=1,2$, two consecutive intersection points between these functions, $\psi_{1}\left(s_{i}^{*}\right)=\psi_{2}\left(s_{i}^{*}\right)=\psi_{i}^{*}>0, i=1,2$. By construction and standard theory of ODEs, the function $\psi(s)=\psi_{1}(s)-\psi_{2}(s)$ does not change sign in the interval $\left[s_{1}^{*}, s_{2}^{*}\right]$ and its derivative $\psi^{\prime}(s)$ has opposite signs at $s_{1}^{*}$ and $s_{2}^{*}$. Then it is clear that at least one of the points $P_{i}^{*}=\left(\psi_{2}\left(s_{i}^{*}\right), w_{2}\left(s_{i}^{*}\right)\right), i=1,2$, belongs to the set $S$. On the other hand, 23. implies that the point $P_{2}(0)=\left(0, w_{2}(0)\right) \in \Sigma_{2} \cap S^{C}$ (where $S^{C}$ means the complement of $S$ ). Hence, it follows from the continuity of the orbit that $\Sigma_{2}$ intersects $\Sigma_{1}$, a contradiction.

Using the fact that the system is autonomous, and the monotonicity of the trajectories in the phase plane, it can be easily proved that only the intersections represented in Fig. 2 are admissible. Moreover, a similar analysis gives a useful estimate of the intersections between the solutions and their translations:

Proposition 3.14. Let $\psi_{1}(s)$ and $\psi_{2}(s)$ be solutions of $(9)$. Then, for any positive constant $\tau$, if we define $\widetilde{\psi}_{2}(s)=\psi_{2}(s+\tau)$, then $I\left(\psi_{1}, \widetilde{\psi}_{2}\right) \leq 2$.

\subsection{Second transformation}

The previous analysis suggests that a convenient transformation in order to disregard the non-energy solutions would be to consider the new variables

$$
\phi(s)=e^{\mu_{+} s} \psi(s) .
$$

After using equation (9) we see that $\phi(s)$ satisfies

$$
\phi_{s s}+2 \sqrt{h^{2} / 4-k} \phi_{s}+e^{\mu_{+}(q-1) s} \phi^{q}=0, \quad \phi>0 \quad \text { for } s<0, \quad \phi(0)=0 .
$$


Remark 3.15. With this change of variables, energy solutions satisfy

$$
\lim _{s \rightarrow-\infty} \phi(s)=C>0
$$

while singular solutions satisfy $\lim _{s \rightarrow-\infty} \phi(s)=+\infty$.

If we restrict ourselves to the case of energy solutions, then we can improve the result concerning the number of intersections. Precisely, we have:

Proposition 3.16. Let $N \geq 3,1<q<(N+2) /(N-2)$, and $0<\lambda<(N-2)^{2} / 4$. Let $\psi_{1}$ and $\psi_{2}$ be solutions of (9)-(10) satisfying (26) after the transformation (24). Then

$$
I\left(\psi_{1}, \psi_{2}\right)=0 \text {. }
$$

Proof. Assume by contradiction that (27) is false and that there are solutions $\psi_{1}$ and $\psi_{2}$ intersecting each other. From Proposition 3.13 it follows that at most one intersection between these functions is possible. Let $\phi_{1}(s)$ and $\phi_{2}(s)$ be obtained by applying transformation 24 to the solutions of $90-10$ given by $\psi_{1}(s)$ and $\psi_{2}(s)$, and set $C_{i}=$ $\lim _{s \rightarrow-\infty} \phi_{i}(s), i=1,2$. Suppose that $C_{1}>C_{2}$. Next, define $\widetilde{\psi}_{2}(s)=\psi_{2}(s+\tau)$, where the constant $\tau=\left(1 / \mu_{+}\right) \log \left(C_{1} / C_{2}\right)$ is chosen in such a way that $\lim _{s \rightarrow-\infty} \phi_{1}(s)=$ $\lim _{s \rightarrow-\infty} \widetilde{\phi}_{2}(s)=C_{1}$ (in particular, if $C_{1}=C_{2}$ then $\tau=0$ ).

It follows by Proposition 3.14 that there exists $s_{*}$ such that $\psi_{1}$ and $\widetilde{\psi}_{2}$ do not intersect for $s<s_{*}$. We assume that in this region $\psi_{1}>\widetilde{\psi}_{2}$, the other case being analogous. (See Fig. 3, which shows the behaviour of $\psi_{1}, \psi_{2}$ and $\widetilde{\psi}_{2}$.)

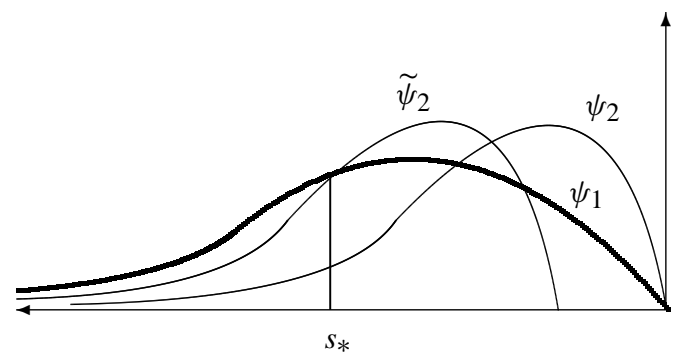

Fig. 3. Solutions and translations of solutions.

Define $g(s)=\phi_{1}(s)-\widetilde{\phi}_{2}(s)$. This function satisfies

$$
g_{s s}+2 \sqrt{h^{2} / 4-k} g_{s}+e^{\mu_{+}(q-1) s}\left(\phi_{1}^{q}-\widetilde{\phi}_{2}^{q}\right)=0,
$$

and moreover

$$
g>0 \text { for } s<s_{*}, \quad \text { and } \quad \lim _{s \rightarrow-\infty} g(s)=\lim _{s \rightarrow-\infty} g^{\prime}(s)=0 .
$$

Integrating equation (28) in the interval $(-\infty, s)$ for some $s<s_{*}$, using 29], we get

$$
g^{\prime}(s)<-\int_{-\infty}^{s} e^{\mu_{+}(q-1) t}\left\{\phi_{1}^{q}(t)-\widetilde{\phi}_{2}^{q}(t)\right\} d t<0 .
$$

And this contradicts 29]. 
The uniqueness result is now a consequence of Proposition 3.16

Proof of Theorem 3.2. Let $u_{1}(r)$ and $u_{2}(r)$ be energy solutions to the problem (6). It follows from Proposition 3.16 that they have to satisfy $I\left(u_{1}, u_{2}\right)=0$, and hence, the solutions are ordered in the interval $(0,1)$. Without loss of generality, we may assume that $u_{2}(r)>u_{1}(r)>0$ for every $r \in(0,1)$. Multiplying by $u_{2}$ the equation corresponding to $u_{1}$, and by $u_{1}$ the equation corresponding to $u_{2}$, and integrating by parts, we get

$$
\int_{B_{1}} u_{1}^{q} u_{2} d x=\int_{B_{1}} u_{2}^{q} u_{1} d x
$$

which is impossible because $u_{2}(r)>u_{1}(r)>0$.

\subsection{Some remarks about the singular solutions}

The previous analysis yields the existence of a unique energy solution, but in the case $k>0$ also the existence of infinitely many singular solutions. Looking at the phase plane, and using some continuity arguments, it is not hard to prove the following properties of singular solutions:

Proposition 3.17. Let $v$ be a singular solution. Then:

- If $u$ is the energy solution, then $I(u, v)=1$. Moreover, $u^{\prime}(1)<v^{\prime}(1)<0$.

- There exists a singular solution $v_{1}$ such that $I\left(v, v_{1}\right)=0$.

- There exists a singular solution $v_{2}$ such that $I\left(v, v_{2}\right)=1$.

\section{References}

[1] Adimurthi, Yadava, S. L.: An elementary proof of the uniqueness of positive radial solutions of a quasilinear Dirichlet problem. Arch. Rat. Mech. Anal. 127, 219-229 (1994) Zbl 0806.35031 MR 1288602

[2] Ambrosetti, A., Brezis, H., Cerami, G.: Combined effects of concave and convex nonlinearities in some elliptic problems. J. Funct. Anal. 122, 519-543 (1994) Zbl 0805.35028 MR 1276168

[3] Ambrosetti, A., Hess, P.: Positive solutions of asymptotically linear elliptic eigenvalue problems. J. Math. Anal. Appl. 73, 411-422 (1980) Zbl 0433.35026 MR 0563992

[4] Baras, P., Goldstein, J.: The heat equation with a singular potential. Trans. Amer. Math. Soc. 294, 121-139 (1984) Zbl 0556.35063 MR 0742415

[5] Berestycki, H., Nirenberg, L.: On the method of moving planes and the sliding method. Bol. Soc. Brasil. Mat. (N.S.) 22, 1-37 (1991) Zbl 0784.35025 MR 1159383

[6] Brezis, H., Dupaigne, L., Tesei, A.: On a semilinear elliptic equation with inverse-square potential. Selecta Math. 11, 1-7 (2005)

[7] Brezis, H., Vázquez, J. L.: Blow-up solutions of some nonlinear elliptic problems. Rev. Mat. Complut. 10, 443-469 (1997) Zbl 0894.35038 MR 1605678

[8] Chaves, M., García Azorero, J.: On uniqueness of positive solutions of semilinear elliptic equations with singular potential. Adv. Nonlinear Stud. 3, 273-288 (2003) Zbl 1071.35053 MR 1971316 
[9] García Azorero, J., Peral, I. Some remarks on bifurcation. Differential Integral Equations 16, 281-302 (2003) Zbl pre02004901 MR 1947954

[10] García Azorero, J., Peral, I.: Hardy inequalities and some critical elliptic and parabolic problems. J. Differential Equations 144, 441-476 (1998) Zbl 0918.35052 MR 1616905

[11] Ghoussoub, N., Yuan, C.: Multiple solutions for quasilinear pdes involving the critical Sobolev and Hardy exponents. Trans. Amer. Math. Soc. 352, 5703-5743 (2000) Zbl 0956.35056 MR 1695021

[12] Gidas, B., Ni, W. M., Nirenberg, L.: Symmetry and related properties via the maximum principle. Comm. Math. Phys. 68, 209-243 (1979) Zbl 0425.35020 MR 0544879

[13] Gidas, B., Spruck, J.: A priori bounds for positive solutions of nonlinear elliptic equations. Comm. Partial Differential Equations 6, 883-901 (1981) Zbl 0462.35041 MR 0619749

[14] Hartman, P.: Ordinary Differential Equations. Birkhäuser (1982) Zbl 0476.34002 MR 0658490

[15] Kwong, M. K., Li, Y.: Uniqueness of radial solutions of semilinear elliptic equations. Trans. Amer. Math. Soc. 333, 339-363 (1992) Zbl 0785.35038 MR 1088021

[16] Rabinowitz, P. H.: Some global results for nonlinear eigenvalue problems. J. Funct. Anal. 7, 487-513 (1971) Zbl 0212.16504 MR 0301587

[17] Rabinowitz, P. H.: On bifurcation from infinity. J. Differential Equations 14, 462-475 (1973) Zbl 0272.35017 MR 0328705

[18] Serrin, J.: A symmetry problem in potential theory. Arch. Rat. Mech. Anal. 43, 304-318 (1971) Zbl 0222.31007 MR 0333220

[19] Terracini, S. On positive entire solutions to a class of equations with singular coefficient and critical exponent. Adv. Differential Equations 1, 241-264 (1996) Zbl 0847.35045 MR 1364003

[20] Whyburn, G. T.: Topological Analysis. Princeton Univ. Press, Princeton, NJ (1964) Zbl 0186.55901 MR 0165476

[21] Yadava, S. L.: Existence and exact multiplicity of positive radial solutions of semilinear elliptic problems in annuli. Adv. Differential Equations 6, 129-154 (2001) Zbl 1064.35060 MR 1799748

[22] Yanagida, E.: Uniqueness of positive radial solutions of $\Delta u+g(r) u+h(r) u^{p}=0$ in $\mathbb{R}^{N}$. Arch. Rat. Mech. Anal. 115, 257-274 (1991) Zbl 0737.35026 MR 1106294 